\title{
Development of an Auditory Rehabilitation Training Programme for Hearing Impaired Adults in Saudi Arabia
}

\author{
Aseel Alkhamees ${ }^{1}$, Karen Bryan ${ }^{2}$ and Merle Mahon ${ }^{3}$ \\ 1. Faculty of Health and Medical Sciences, King Saud University, Riyadh 11352, Kingdom of Saudi Arabia \\ 2. Faculty of Health and Wellbeing, Sheffield Hallam University, Sheffield S10 2BP, UK \\ 3. UCL Developmental Science Research Department, Division of Psychology and Language Sciences, University College London, \\ London WC1N $1 P F$, UK
}

\begin{abstract}
Hearing impairment is one of the most common sensory disabilities, affecting millions of people worldwide. The current management of acquired hearing impairment in adults is usually in the form of hearing aids. An auditory rehabilitation programme may or may not be provided although literature suggests that such programmes may enhance hearing aid benefits. This study was conducted in an Arabic-speaking country, specifically Saudi Arabia, and is the first to develop an auditory rehabilitation intervention programme in the Arabic language that focuses on working age adults who are new hearing aid users. Randomised intervention study design. Thirty-five participants with mild to severe acquired hearing loss (intervention group $n=18$, control group $n=17$ ). Significant changes were found in the performance of the intervention group in the Minimal Audible Capabilities (MAC) test, a lip reading test and the Glasgow Hearing Aid Benefit Profile (GHABP) questionnaire when compared with the control group. No significant difference was found in speech test scores between the two groups. A short individualised auditory rehabilitation intervention programme can yield a significant improvement in the performance of hearing impaired adults by enhancing the benefits of hearing aid use.
\end{abstract}

Key words: Hearing loss, hearing aid, auditory rehabilitation, speech test, Minimal Audible Capabilities (MAC) test, lip reading test, Glasgow Hearing Aid Benefit Profile (GHABP) questionnaire, Saudi Arabia.

\section{Introduction}

Hearing impairment has become one of the most prevalent chronic disabilities affecting the world's population. The number of hearing impaired people in the world is increasing gradually every year due to global population growth and longer life expectancy. According to the World Health Organization (WHO), it was estimated in 1995 that there were 120 million people suffering from a hearing impairment, which equates to approximately $2.1 \%$ of the global population. By the year 2000 the number of sufferers had more than doubled to 250 million people worldwide. This trend continued such that in 2005 the estimate rose to 278 million people, and by 2013 it was estimated that the number had increased to 360 million people with a

Corresponding author: Aseel Alkhamees, assistant professor, research field: audiology. disabling hearing impairment worldwide, accounting for 5\% of the world's population [1-3].

Saudi Arabia has the third highest population of deaf people in the Middle East region, after Algeria and Iraq. There are $3.55 \%$ deaf people in the general population, according to World Federation of the Deaf [4]. In Saudi Arabia this number is high because of the frequent practice of consanguineous marriage, which increases the chance of transmitting inherited conditions such as certain types of hearing impairment [5]. The study reported here focuses on adults who suffer from a hearing impairment in Riyadh city, the capital of Saudi Arabia, and investigates the benefits of using hearing aids in combination with an auditory rehabilitation intervention programme.

The effects of a hearing impairment can impact on an individual's life in many different ways, for instance affecting their communication skills, their mental 
health and social engagement and their overall quality of life $[6,7]$. The current rehabilitation model available for managing acquired hearing impairment in adults is to use an auditory amplification device, usually a hearing aid, in conjunction with a programme of auditory rehabilitation. The definition of auditory rehabilitation has not changed substantially over the years, it is, as Hull [8] defined it "an attempt at reducing the barriers to communication resulting from hearing impairment, and facilitating adjustment relative to the possible psychosocial, occupational and educational impact of the auditory deficit". Research in the USA and Western Europe suggests that a programme of auditory rehabilitation enhances the benefit of a hearing aid [9-12]. There are no auditory rehabilitation programmes in place in Saudi Arabia, and no research has been conducted to investigate rehabilitation outcomes in Arabic-speaking countries or in Islamic cultures. This study is the first to focus on hearing impaired adults who are new hearing aid users in Saudi Arabia.

The first aim of the study was to develop an auditory rehabilitation intervention programme that includes home training tasks. The second aim was to investigate differences in terms of the perception of benefits from hearing aid use in the auditory performance of new hearing aid users who receive either an auditory rehabilitation intervention programme or a standard package of care.

\section{Methods}

\subsection{Subjects}

Thirty-five participants were recruited from a local audiology clinic in one of the main hearing aid centres in Riyadh city. All participants were new hearing aid users who had bilateral mild to severe sensorineural hearing loss; they were aged between 18 and 60 years with a mean age of 42.8 years $(\mathrm{SD}=11.75)$. All the participants were seen by an ENT specialist, and performed pure-tone audiometry (PTA) using a GN Otometric MADSEN audiometer with circum-aural headphones and bone conductor. Participants were sequentially randomised according to their age and the severity of their hearing loss into two groups: 17 participants were in the Standard Care (SC) group (the control group), and 18 participants were in the Auditory Rehabilitation (AR) group (the intervention group). Table 1 summarises demographic and hearing status characteristics of the two participant groups.

\subsection{Outcome Measures}

Four outcome measures were used to assess the effects of the auditory rehabilitation programme on the use of hearing aids. These were:

\subsubsection{Speech Test}

The Arabic speech test developed by Ashoor and Prochazka [13], consisting of phonetically balanced monosyllabic Arabic words was used. The test consists of six equivalent lists, each containing 20 words.

2.2.2 Minimal Audible Capabilities (MAC) Test Battery

The Minimal Audible Capabilities test battery was developed by Owens et al. [14]. The test is specifically aimed at assessing the hearing abilities of profound acquired hearing impaired adults who use hearing aids or cochlear implants. Arabic translated modified version of the MAC test [15] was used in the study. However, for the purpose of this study, focusing on adults with acquired mild to severe sensorineural hearing loss, certain sub-tests were selected. These were: Noise/Voice test, Accent test, Everyday Sentences test, and High Context Sentences test.

\subsubsection{Lip Reading Test}

The Arabic lip reading test that was used in the study was developed by King Abdulaziz University Hospital [16]. The test consists of words and sentences categorised into four types. These are: Familiar Words, Vowels, Consonants, and Long Sentences.

\subsubsection{Glasgow Hearing Aid Benefit Profile (GHABP)} Questionnaire

An Arabic translated version of the GHABP questionnaire was used in the study [17]. The 
Table 1 Participants' demographic and hearing status characteristics.

\begin{tabular}{lll}
\hline & $\begin{array}{l}\text { Standard Care (SC) group } \\
(\mathrm{n}=17)\end{array}$ & $\begin{array}{l}\text { Auditory Rehabilitation (AR) group } \\
(\mathrm{n}=18)\end{array}$ \\
\hline Age & $43.06(13.18)$ & $42.56(10.6)$ \\
Mean (SD) & 8 & 9 \\
Age range 18-40 & 9 & 9 \\
$\quad$ Age range 41-60 & 8 & 7 \\
\hline Gender & 9 & 11 \\
$\quad$ Female & 3 & 3 \\
$\quad$ Male & 11 & 11 \\
\hline Duration of hearing loss & 3 & 4 \\
$\quad<1$ year & & 9 \\
1-5 years & 9 & 1 \\
$\quad>$ 5 years & - & 8 \\
\hline Degree of hearing loss & 8 & 5 \\
Mild to moderate & 8 & 1 \\
Moderate to severe & 1 & 12 \\
Severe & 8 &
\end{tabular}

questionnaire consists of two parts: 1) four pre-specified listening situations that are common for the hearing impaired person in everyday life; and 2) four nominated listening situations that the hearing impaired person specifies according to their importance in their everyday communication. Each of these two parts is assessed separately in two sections: A) without the hearing aid, which assesses Initial Disability and Handicap; and B) with the hearing aid, which assesses Use, hearing aid Benefit, Residual Disability and Satisfaction.

All the four outcome measures were administered by an audiologist and all the assessment data for the SC and AR groups were kept in private coded files to ensure that the researcher who delivers the auditory rehabilitation intervention programme was as blind as possible to the outcome results.

\subsection{Procedures}

The protocol for this study was approved by the Saudi Arabia Ministry of Health and permission to conduct the study was obtained from the University of
Surrey Ethics Committee. Ethical permission was also granted by the hearing aid centre where the participants were recruited from.

The participants were sequentially randomised according to their age and the severity of their hearing loss into two groups: a standard care (SC) group and an auditory rehabilitation (AR) group. Figures 1 and 2 summarise the SC and AR groups' trial procedure.

At their initial appointment (appointment 1) for hearing aid evaluation all participants were asked to complete the without hearing aid section of the self-assessment GHABP questionnaire. Thereafter the lip reading test was administered in a quiet room with the audiologist sitting face to face and one metre away from the participant. This was followed by the speech test (Unaided), performed via a live voice with visual cues presented at $60 \mathrm{~dB}(\mathrm{~A})$ measured by a sound level meter. The participant was asked to sit in the centre of a quiet room and informed that she/he would hear 20 words. After each word she/he had to repeat the word that they had heard. 


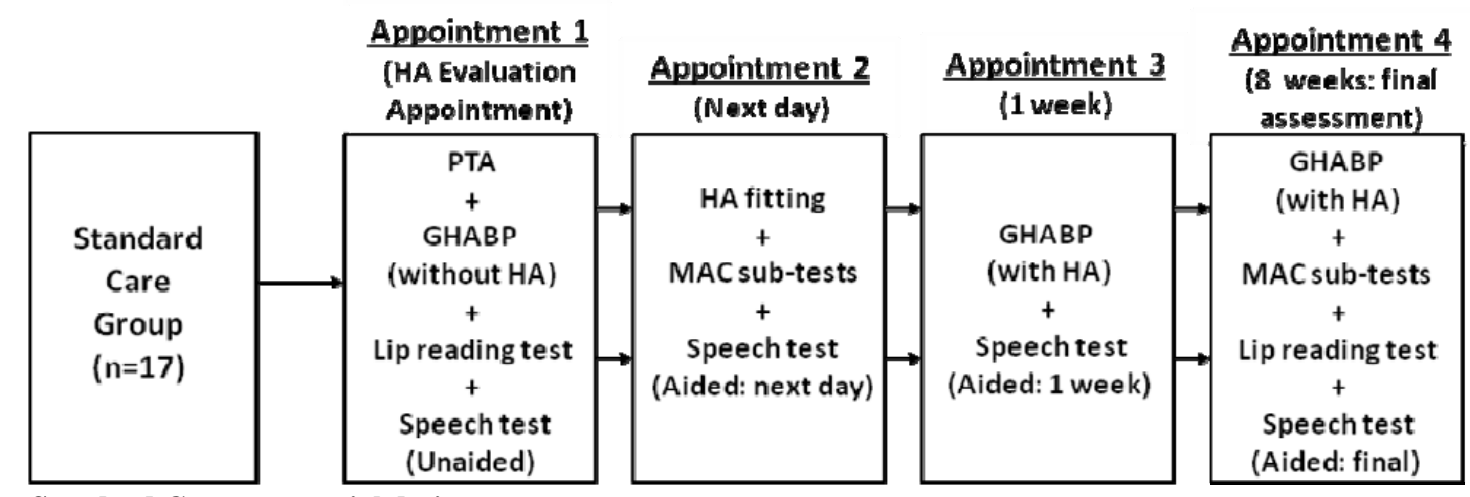

Fig. 1 Standard Care group trial design.

(PTA: pure-tone auditometry, HA: hearing aid)

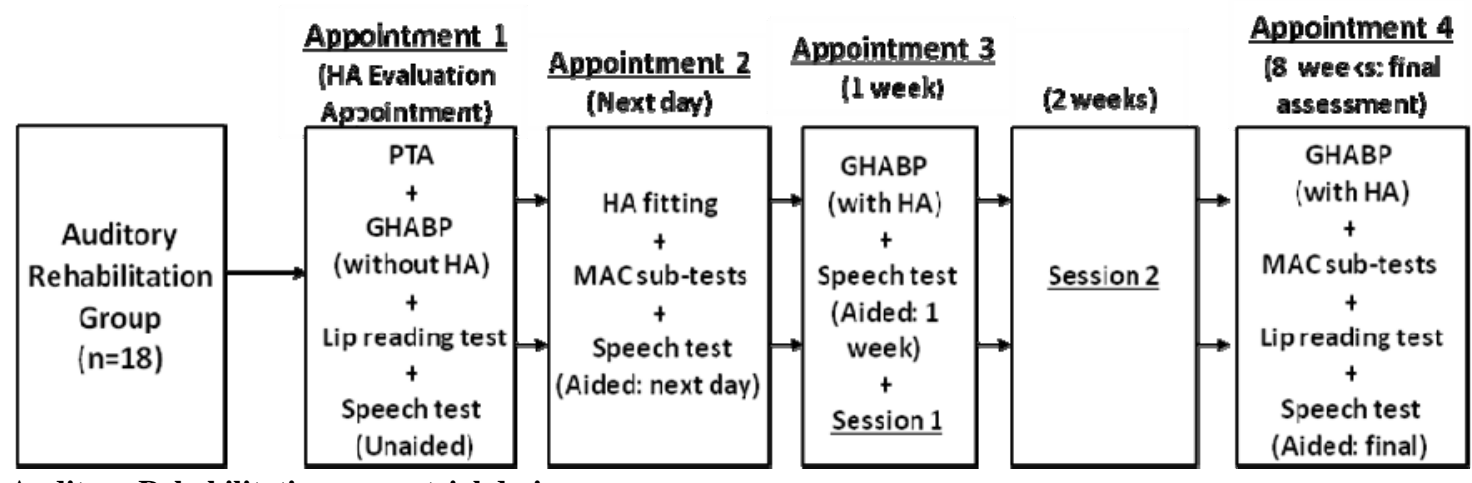

Fig. 2 Auditory Rehabilitation group trial design.

(PTA: pure-tone auditometry, HA: hearing aid)

At the next appointment on the following day (appointment 2), after the participants had been fitted with digital hearing aids, the MAC sub-tests were administered in a sound-treated booth with minimum visual and auditory distraction, using a GN Otometric MADSEN audiometer. Each participant was seated at the same distance, approximately one metre away and at $45^{\circ}$ azimuths from a matched loudspeaker system. All the test stimuli were live voice words, except for the Noise/Voice sub-test where pink noise was used and presented simultaneously with live voice stimuli in the noise condition. All the stimuli were presented according to the individual participants' most comfortable loudness (MCL) level, which had been identified prior to the test. For the Noise/Voice sub-test in the noise condition the signal-to-noise ratio was +10 $\mathrm{dB}$ according to the MCL of the participants. After that, the speech test (Aided: next day) was performed in the same way as before.
In appointment 3, which took place one week after the participants had begun using their hearing aids, the participants were asked to complete the with the hearing aid section of the GHABP questionnaire and perform another speech test (Aided: 1 week).

\subsection{Group Trial}

\subsubsection{Standard Care (SC) Group}

After the participants completed the with the hearing aid section of the GHABP questionnaire and performed the speech test (Aided: 1 week) during appointment 3, they were asked to attend for another follow-up appointment (appointment 4) eight weeks after their enrolment in the study in order to carry out a final assessment. During this assessment they completed the with the hearing aid section of the GHABP questionnaire, four sub-tests from the MAC battery, and repeated the lip reading test and speech test (Aided: final). Also, each participant was advised to 
contact the hearing aid centre if any further assistance was needed.

\subsubsection{Auditory Rehabilitation (AR) Group}

For the AR group, during appointment 3, after completing the with the hearing aid section of the GHABP questionnaire and performing the speech test (Aided: 1 week), the participants had a one-hour intervention session of auditory rehabilitation that focused on education, information and listening training. Afterwards, the participants were given a follow-up appointment the next week for a second intervention session lasting one hour, which focused on hearing strategies and listening training. The auditory rehabilitation intervention programme was designed to be administered face to face in two individual sessions. Individual sessions were preferred rather than group sessions due to the nature of Saudi Arabian society, which is more conservative and protective of personal privacy, and also rigidly segregated between men and women. The programme [18] was based on common content from published group rehabilitation programmes [9, 11, 19, 20] adapted for a shorter, individualised delivery with targeted home practice.
Table 2 summarises the components of the auditory rehabilitation intervention programme for each session. Finally, the participants were given another follow-up appointment (appointment 4) eight weeks after their enrolment in the study, which followed the same procedure as appointment 4 for the SC group.

\section{Analysis}

All the statistical analyses of the data were conducted using SPSS version 19. The data from the speech test, the MAC sub-tests and the lip reading test were statistically analysed using $t$-tests to determine whether there were any significant differences between the SC and AR groups in terms of the participants' performance. The GHABP questionnaire was analysed using a Mann-Whitney U-test to detect whether there was any significant difference between the performances of the two groups.

\section{Results}

\subsection{Speech Test}

The SC and AR groups' performance in the speech test at the final assessment appointment 4 (Aided: final)

Table 2 Components of auditory rehabilitation intervention programme for each session.

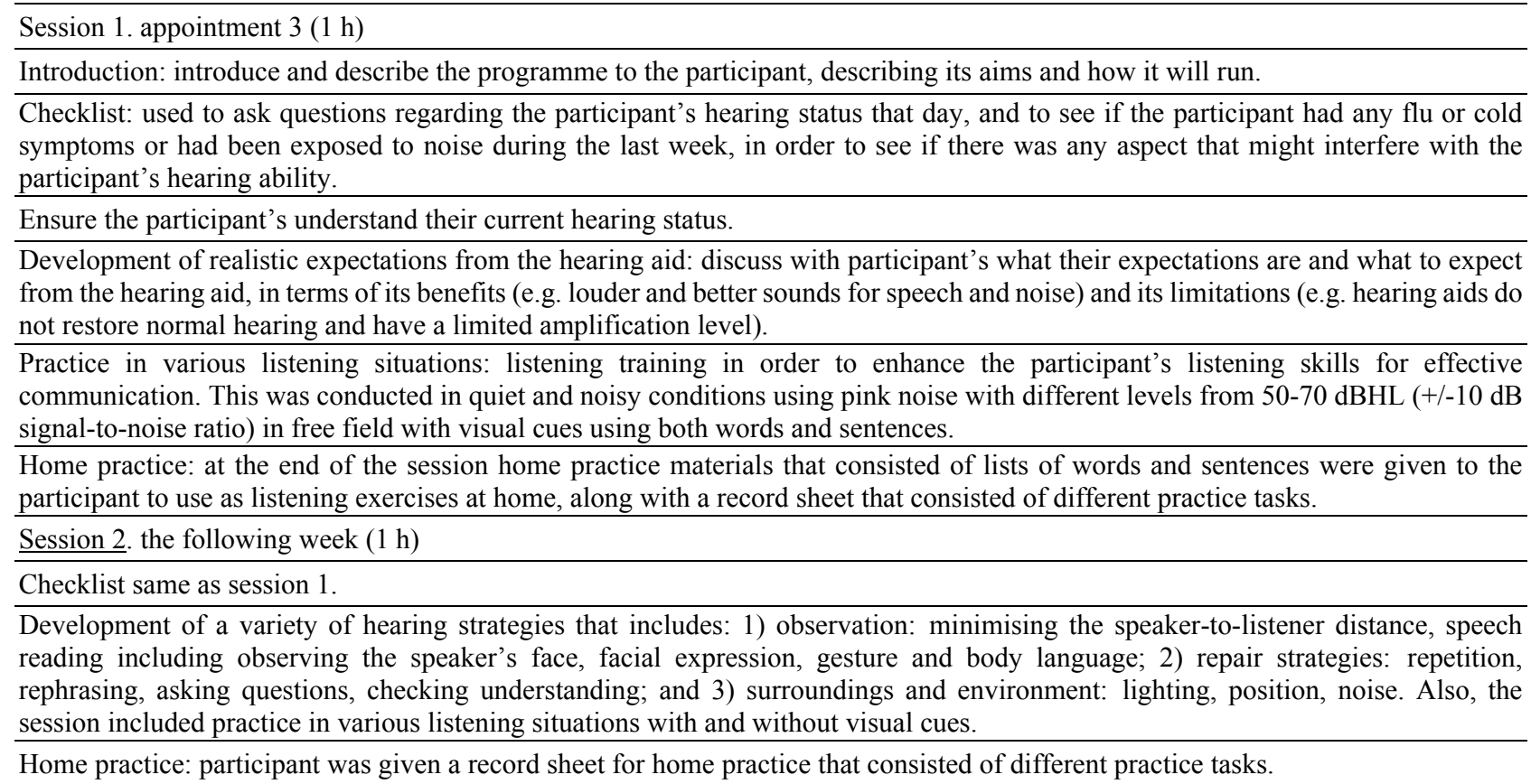


Table 3 Summary of $\boldsymbol{t}$-test results for speech test at final assessment.

\begin{tabular}{ll}
\hline Speech test & $t$-tests $P$-value \\
\hline Unaided (appointment 1) & 0.152 \\
Aided: next day (appointment 2) & 0.564 \\
Aided: 1 week (appointment 3) & 0.585 \\
\hline
\end{tabular}

was compared with their previous performances (appointment 1, 2, 3) in order to detect any changes during this period. Independent sample $t$-tests revealed that there were no statistically significant differences $(P>0.05)$ between the $\mathrm{SC}$ and AR groups for the speech test at the final assessment. Table 3 summarises $t$-tests findings for speech test.

\subsection{MAC Sub-tests}

The SC and AR groups' performance in the MAC test at the final assessment was compared with their baseline performances during appointment 2 . Independent sample $t$-tests showed that for the MAC Noise/Voice test $P=0.038$, for the MAC Accent test $P$ $\leq 0.001$, for the MAC Everyday Sentence $P=0.010$, for the MAC High Context Sentences: Last Word $P=$ 0.036, and for the MAC High Context Sentences: Whole Meaning $P=0.014$. Therefore, there was a significant difference $(P<0.05)$ between the SC and AR performances in the MAC test at the final assessment in favour of the AR group.

\subsection{Lip Reading Test}

The performances of the SC and AR groups in the lip reading tests at the final assessment were compared with their baseline performances during appointment 1 . Independent sample $t$-tests showed that for lip reading Familiar Words $P<0.001$, for lip reading Vowels $P=$ 0.002 , for lip reading Consonants $P<0.001$, and for lip reading Long Sentences $P<0.001$. From these results it is possible to conclude that there was a statistically significant difference $(P<0.05)$ between the SC and AR groups' performance in the lip reading test at the final assessment, in favour of the AR group.

\subsection{GHABP Questionnaire}

The results showed that the total number of situations was 17; four situations were pre-specified, and 13 situations were nominated by the participants. The number of respondents varied between each situation, depending on what each participant specified and how important and relevant the situations were to their everyday communication. Table 4 shows the number of respondents for each situation.

Three situations were chosen by the majority of participants. These were: situation 6: Family gatherings (nominated by 29 participants), situation 12 : Using mobile/phone and situation 14: At work (nominated by 24 participants). These three situations, together with the four pre-specified situations (situation 1, situation 2, situation 3 and situation 4) will be referred to hereafter as the seven common situations; all have a sufficient number of participant respondents to allow statistical comparisons between the SC and AR groups. Therefore, Mann-Whitney U-tests were performed to determine whether there were any significant differences between the participants' performances in the SC and AR groups. However, insufficient numbers of participant respondents chose the remaining ten situations and therefore no statistical comparisons between the SC and AR groups could be performed for those situations.

\subsection{GHABP: Without the Hearing Aid}

This part of the questionnaire assesses the participants' Initial Disability and Handicap. This section was completed just once, before the participants received their hearing aids. Mann-Whitney U-tests were performed for the seven common situations to compare the Initial Disability and Handicap between the SC and AR groups. Results show no statistically significant differences $(P>0.05)$ between the SC and AR groups in terms of the participants' Initial Disability 
Table 4 Number of respondents for each GHABP situation.

Situation

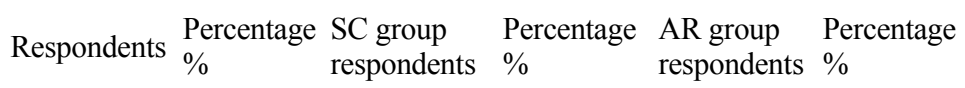

\begin{tabular}{|c|c|c|c|c|c|c|}
\hline $\begin{array}{l}\text { Situation } 1 \text { (Sit 1): Listening to the television with other } \\
\text { family or friends when the volume is adjusted to suit } \\
\text { other people }\end{array}$ & 35 & $100 \%$ & 17 & $100 \%$ & 18 & $100 \%$ \\
\hline $\begin{array}{l}\text { Situation } 2 \text { (Sit 2): Having a conversation with one other } \\
\text { person when there is no background noise }\end{array}$ & 35 & $100 \%$ & 17 & $100 \%$ & 18 & $100 \%$ \\
\hline $\begin{array}{l}\text { Situation } 3 \text { (Sit 3): Carrying on a conversation in a busy } \\
\text { street or shop }\end{array}$ & 35 & $100 \%$ & 17 & $100 \%$ & 18 & $100 \%$ \\
\hline $\begin{array}{l}\text { Situation } 4 \text { (Sit } 4 \text { ): Having a conversation with several } \\
\text { people in a group }\end{array}$ & 35 & $100 \%$ & 17 & $100 \%$ & 18 & $100 \%$ \\
\hline Situation 5 (Sit 5): Weekly meetings & 17 & $48.5 \%$ & 11 & $64.7 \%$ & 6 & $33.3 \%$ \\
\hline Situation 6 (Sit 6): Family gatherings & 29 & $82.8 \%$ & 15 & $88.2 \%$ & 14 & $77.7 \%$ \\
\hline Situation 7 (Sit 7): Quran classes and religious lectures & 3 & $8.5 \%$ & 1 & $5.8 \%$ & 2 & $11.1 \%$ \\
\hline Situation 8 (Sit 8): Meeting new people & 4 & $11.4 \%$ & 2 & $11.7 \%$ & 2 & $11.1 \%$ \\
\hline Situation 9 (Sit 9): At lectures & 9 & $25.7 \%$ & 4 & $23.5 \%$ & 5 & $27.7 \%$ \\
\hline $\begin{array}{l}\text { Situation } 10 \text { (Sit 10): Restaurants with noisy } \\
\text { environments }\end{array}$ & 2 & $5.7 \%$ & 1 & $5.8 \%$ & 1 & $5.5 \%$ \\
\hline Situation 11 (Sit 11): At lectures while sitting at the back & 1 & $2.8 \%$ & 1 & $5.8 \%$ & - & - \\
\hline Situation 12 (Sit 12): Using mobile/phone & 24 & $68.5 \%$ & 10 & $58.8 \%$ & 14 & $77.7 \%$ \\
\hline Situation 13 (Sit 13): At mosque & 11 & $31.4 \%$ & 6 & $35.2 \%$ & 5 & $27.7 \%$ \\
\hline Situation 14 (Sit 14): At work & 24 & $68.5 \%$ & 10 & $58.8 \%$ & 14 & $77.7 \%$ \\
\hline Situation 15 (Sit 15): With children at home & 1 & $2.8 \%$ & 1 & $5.8 \%$ & - & - \\
\hline Situation 16 (Sit 16): Talking in the distance & 8 & $22.8 \%$ & 3 & $17.6 \%$ & 5 & $27.7 \%$ \\
\hline Situation 17 (Sit 17): With head scarf & 7 & $20 \%$ & 3 & $17.6 \%$ & 4 & $22.2 \%$ \\
\hline
\end{tabular}

and Handicap. Figure 3 shows the average difference between the $\mathrm{SC}$ and $\mathrm{AR}$ groups in terms of Initial Disability and Handicap for the four pre-specified situations. The $\mathrm{Y}$-axis represents the scale according to the GHABP questionnaire and the $\mathrm{X}$-axis represents the four pre-specified situations.

\subsection{GHABP: With the Hearing Aid}

This part of the questionnaire assesses the participants' hearing aid Use, hearing aid Benefit, Residual Disability and Satisfaction. This section of the questionnaire was completed twice, once after one week of wearing the hearing aid (during appointment 3) and again after eight weeks of wearing the hearing aid (at appointment 4). The data was analysed by comparing the participants' performances at eight weeks with their performance after one week. Mann-Whitney U-tests were performed for the seven common situations to compare the changes in the participants' performances according to their hearing aid Use, hearing aid Benefit, Residual Disability, and Satisfaction scores. The results revealed statistically significant differences in the participants' performance in situation 1 for Use $(P=0.035)$, hearing aid Benefit $(P=0.040)$ and Satisfaction $(P=0.046)$. In situation 2 a statistically significant difference was only found for hearing aid Benefit $(P=0.026)$. In situation 3 a statistically significant difference was found for hearing aid Benefit ( $P=0.001)$, Residual Disability ( $P$ $=0.004)$ and Satisfaction $(P \leq 0.001)$. In situation $4 \mathrm{a}$ statistically significant difference was found in the participants' performance for Residual Disability $(P=$ $0.025)$ and Satisfaction $(P=0.045)$. In situation 6 , only Satisfaction showed a statistically significant difference $(P=0.029)$. In situation 12 a statistically significant difference was found in the participants' performance for hearing aid Benefit $(P \leq 0.001)$, Residual Disability $(P=0.009)$ and Satisfaction $(P=0.003)$. 

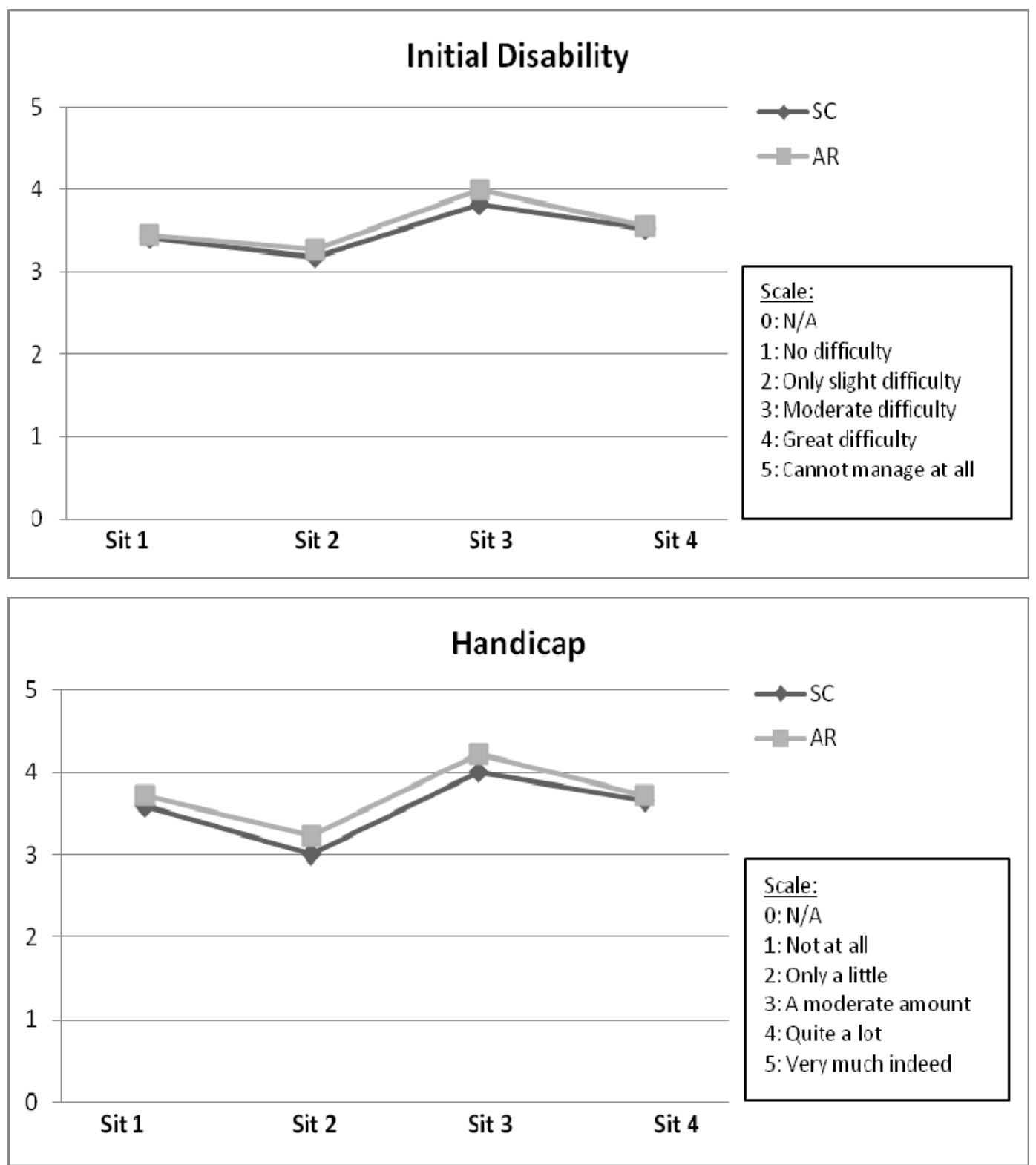

Fig. 3 Initial Disability and Handicap in the four pre-specified situations without the hearing aid.

Finally, in situation 14 there were no statistically significant differences $(P>0.05)$ between the SC and AR groups for Use, hearing aid Benefit, Residual Disability or Satisfaction.

The following figures show the average difference between the SC and AR groups in terms of Use, hearing aid Benefit, Residual Disability and Satisfaction for the four pre-specified situations after eight weeks of wearing the hearing aid. The Y-axis represents the scale according to the GHABP questionnaire and the $\mathrm{X}$-axis represents the four pre-specified situations

\section{Discussion}

The targets of the auditory rehabilitation intervention programme are to reduce the amount of disability and handicap presented by the participants' auditory impairments, improving and optimising the use of and benefit from hearing aids and minimising activity restriction in terms of everyday communication.

The outcome measures were conducted at the start of the study, to obtain a baseline measure for each 

Hearing Impaired Adults in Saudi Arabia
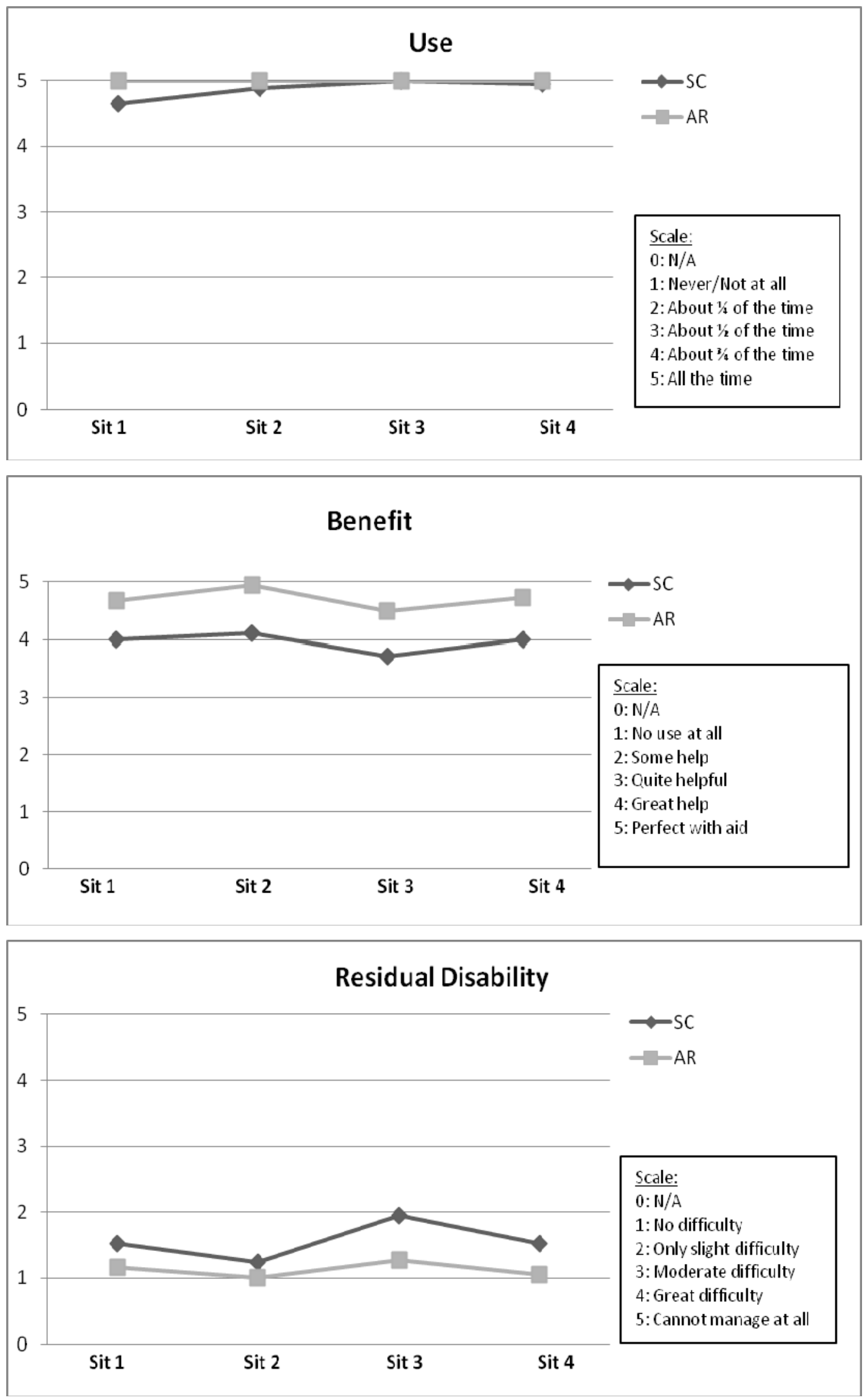


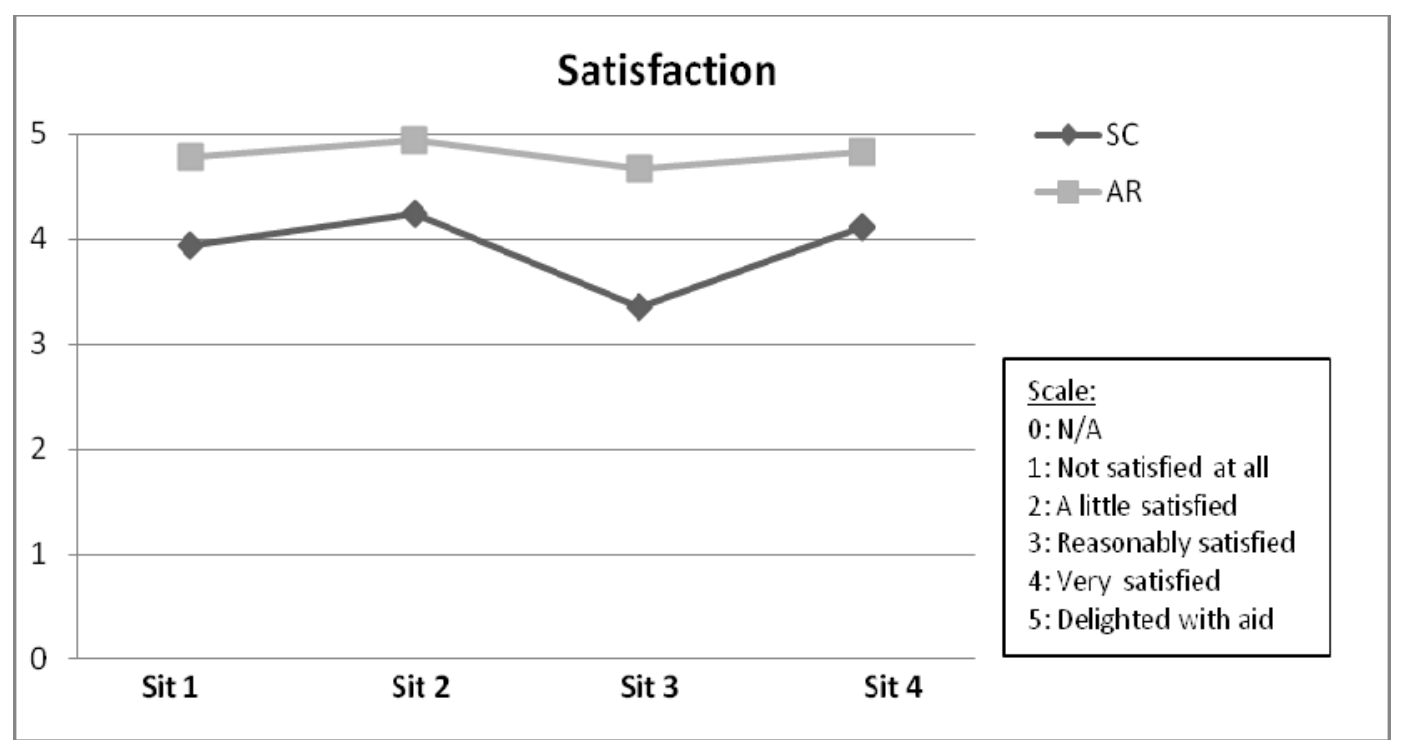

Fig. 4 Use, hearing aid Benefit, Residual Disability and Satisfaction after eight weeks of wearing the hearing aid in the four pre-specified situations.

participant, and again at a final assessment eight weeks from the time of enrolment in the study. The only exception was the speech test, which was assessed four times. The rationale for having the final assessment eight weeks after being fitted with the hearing aid was to ensure that the participants' performance was stable and not exaggerated, since a number of studies have found that participants' performance three weeks after hearing aid fitting tends to be better than when the evaluation is performed three to six months later. This is known as the 'honeymoon' or 'halo' effect, referring to an initial increase in the hearing aid user's reaction towards the benefit they receive from the hearing aid $[21,22]$. To avoid this effect several studies have suggested that final assessments should be performed between six and eight weeks after being fitted with the hearing aid because the hearing aid user's performance tends to be stable during this period of time [23, 24].

The speech test results showed that there were no statistically significant differences between the SC and AR groups at the final assessment. This may indicate that both groups had improved by around the same amount. A possible reason for this could be that all the participants in the current study used digital hearing aids, they might be expected to show an improvement and achieve a better score in their speech test at their final assessment (appointment 4) when compared to their performances at the first hearing aid evaluation appointment 1 (Unaided), appointment 2 (Aided: next day) and their performance at appointment 3 (Aided: 1 week). There is evidence showing that digital hearing aids provide immediate benefit to speech recognition $[25,26]$. Although the AR group showed slightly better performance than the SC group, the difference between the two groups did not reach statistical significance. One possible reason for this might be that the sample size was too small to show such an effect between the two groups.

The MAC sub-tests results showed that there was a significant difference between the $\mathrm{SC}$ and $\mathrm{AR}$ performances at the final assessment. The AR group showed a significant improvement in speech recognition in noisy and quiet situations since they scored significantly higher in the Noise/Voice test, in identifying the stressed word in the Accent test, and in understanding and recognising speech in the Everyday Sentence and High Context Sentence tests (understanding the overall meaning and repeating the last word). This confirms what Kricos [10] and Boothroyd [27] reported, i.e. that auditory training involving the practice of a variety of listening situations as part of an auditory rehabilitation 
intervention programme (with home practice tasks as well) will lead to enhanced speech recognition and better perception in various listening situations. Similarly, a study conducted by Sweetow and Sabes [28] developed a home-based computerised listening and communication enhancement programme for hearing impaired adults which showed that participants who used the training programme improved significantly in terms of both subjective and objective measures.

The lip reading test results showed that there was a significant difference between the SC and AR group performances at the final assessment in favour of the AR group. This improvement by the AR group confirms that combined auditory and visual perception can facilitate enhanced performance in speech understanding over and above relying on auditory input alone. It is interesting to note that existing research on lip reading supports the current study's findings. Erber [29] reported that it is better to combine auditory and visual perception than to rely on either auditory or visual perception alone. Moreover, there are numerous studies showing that hearing impaired individuals tend to have higher scores in word recognition and sentence identification when they listen through their hearing aid and lip read at the same time than when they perceive by listening alone [30-32]. More recently, Dalebout [33] noted that many studies have proved that combining what one sees with what one hears can improve the understanding of speech by more than $30 \%$ in difficult listening situations. Barnett [34] also reported that focusing on non-auditory strategies such as lip movement, facial expression and body posture helps to improve speech perception. Chen and Hazan [35] investigated the factors that affected audio-visual speech perception and found that there was a strong positive correlation between visual input and speech reading performance. This finding suggests that concentrating on both visual and auditory input enhances individual speech perception. In our study, hearing strategies made up part of the auditory rehabilitation intervention programme, being highlighted in the second session.

Walden et al. [36] found that the groups who received two weeks of 50-min auditory rehabilitation sessions in addition to auditory or visual consonant training programmes showed a significant increase in their consonant recognition performance when compared with the group who received auditory rehabilitation alone. The current study's findings suggest that the participants who received two sessions of an auditory rehabilitation intervention programme (the AR group), along with listening training with and without visual cues and home practice tasks, showed significantly better scores in the lip reading test when compared with the group who did not receive the rehabilitation programme (the SC group). This finding suggests that listening training focusing on both auditory and visual elements is useful in improving the performance of hearing impaired individuals in speech understanding. Also, it is suggested that short customised individualised auditory rehabilitation programmes are likely to be less time consuming and less costly for their delivery than substantially longer group auditory rehabilitation programmes.

The GHABP questionnaire results found that there was a total of 17 listening situations, of which four were the pre-specified listening situations and 13 were nominated listening situations as illustrated in Table 4. These situations varied according to the numbers of respondents, as each participant nominated their own situations according to their importance in that person's everyday communication. The fact that the number of respondents varied between the situations negatively affects the potential for comparison between the SC and AR groups. There are situations where the number of respondents is low, and some situations where there are no respondents in one group when compared to the other (e.g. situations 11 and 15). For this reason it was not possible to perform direct statistical comparisons between the $\mathrm{SC}$ and $\mathrm{AR}$ groups. However, statistical comparisons were conducted for 
the most common situations with a high number of respondents, allowing for meaningful comparison between the $\mathrm{SC}$ and $\mathrm{AR}$ groups.

On the without the hearing aid section of the questionnaire the results showed that there were no statistically significant differences between the SC and AR groups in terms of the Initial Disability and Handicap. This indicates that the results were similar for both groups, as the median score for Initial Disability ranged between 3 and 4 and the Handicap was 4 according to the questionnaire scale for both groups. These results were expected as all the participants in both groups had similar age profiles and configuration of hearing loss. Similarly, the results from the without the hearing aid section in Munro and Lutman's study [37], which also used the GHABP questionnaire as a self-report measure, were found to be compatible with the current study as they showed a moderate amount of Initial Disability and Handicap among the two study groups, with no statistically significant difference detected between them [17].

The with the hearing aid section compared the Use, hearing aid Benefit, Residual Disability, and Satisfaction scores of the participants in the SC and AR groups in the seven common situations at final assessment (eight weeks) with their performance at appointment 3 (1 week). The results indicate that the AR group showed better performance and statistically significant improvements in certain parameters of the GHABP, i.e. Use, hearing aid Benefit, Residual Disability or Satisfaction, in certain situations but not in others. In terms of the other parameters that did not show any significant differences between the two groups, the AR group generally showed slightly higher scores than the SC group, but the difference between the performances was not strong enough to reach statistical significance. This might be explained by the fact that the participants in the SC group were satisfied with the use of their hearing aids in certain situations to a level that was the same as the satisfaction of the AR group, especially since all the participants in the study were using digital hearing aids. In Wood and Lutman's study [25], no significant difference was found in self-reported benefit using the GHABP questionnaire and the Abbreviated Profile of Hearing Aid Benefit when comparing between analogue and digital hearing aids $[17,38,39]$.

Munro and Lutman [37] found that $90 \%$ of the study sample wore their hearing aids when they experienced difficult listening situations. In comparison with the current study findings, it was found that the Use of the hearing aid was statistically significant only in situation 1, while in the other situations there were no statistically significant differences found between the SC and AR groups. This indicates that all the participants in the study used their hearing aids effectively and within the same range of use. Kemker and Holmes [40] also used GHABP questionnaire, and found that that participants younger than 66 years old who had received post-fitting orientation had significantly higher means for their Satisfaction scores than those who did not receive any hearing aid orientation [17]. In comparison with the current study, the AR group, who received two post-fitting sessions of a short individualised auditory rehabilitation intervention programme, performed significantly higher in Satisfaction than the SC group across the seven common situations except in situations 2 and 14, where they performed better but the difference did not reach significance.

\section{Conclusions}

The results of this study have added to the evidence that auditory rehabilitation programmes can enhance the benefits of hearing aid usage for adults with acquired hearing difficulties. Also that short individualised auditory rehabilitation intervention programme of two sessions, once per week for one hour, including individually focused home training tasks, can yield a significant improvement in the report of the benefits of hearing aid use by Saudi Arabian hearing impaired adults. Also, short individualised 
auditory rehabilitation programmes are likely to be less time consuming and less costly to deliver than longer group auditory rehabilitation programmes, but they appear to have similar results to the longer programmes. The study provides insights into the effects of an auditory rehabilitation intervention programme on Saudi Arabian adults of working age living in Riyadh, and strongly suggests that this is an appropriate strategy for intervention delivery in Saudi Arabia. The results from this study also provide initial data from a set of assessment adapted for use in Arabic, which will be useful for future researchers working in Saudi Arabia.

\section{References}

[1] World Health Organisation (WHO), 1995. Prevention of Hearing Impairment, Resolution of the 48th World Health Assembly, Geneva, WHA 48.9.

[2] World Health Organisation (WHO), 2010. Deafness and Hearing Impairment, Fact Sheet N 300, Media Centre. Retrieved May 27, 2010, from http://www.who.int/mediacentre/factsheets/fs300/en/inde x.html.

[3] World Health Organisation (WHO), 2013. Deafness and Hearing Loss, Fact Sheet N 300, Media Centre. Retrieved April 6, 2013, from http://www.who.int/mediacentre/factsheets/fs300/en/.

[4] World Federation of the Deaf (WFD), 2008. Global Survey Report WFD Interim Regional Secretariat for the Arab Region (WFD RSAR) by the World Federation of the Deaf and the Swedish National Association of the Deaf.

[5] Zakzouk, S. 2002. "Consanguinity and Hearing Impairment in Developing Countries: a Custom to be Discouraged.” Journal of Laryngology and Otology 116: 811-6.

[6] Dalton, D. S., Cruickshanks, K. J., Klein, B. E. K., Klein, R., Wiley, T. L., and Nondahl, D. M. 2003. "The Impact of Hearing Loss on Quality of Life in Older Adults." The Gerontologist 43: 661-8.

[7] Weener, D. J., Zacharek, M. A., and Malani, P. N. 2010. "Evaluation and Management of Hearing Loss in Older Adult." Clinical Geriatircs 18: 20-6.

[8] Hull, R. H. 1982. Rehabilitative Audiology. University of Michigan: Grune \& Stratton.

[9] Abrams, H., Hnath-Chisolm, T., Guerreiro, S., and Ritterman, S. 1992. "The Effects of Intervention Strategy on Self-perception of Hearing Handicap." Ear and
Hearing 13: 371-7.

[10] Kricos, P. B. 1997. "Audiological Rehabilitation for the Elderly: a Collaborative Approach.” The Hearing Journal 50: 10-9.

[11] Preminger, J. E. 2003. "Should Significant Others be Encouraged to Join Adult Group Audiologic Rehabilitation Classes?" Journal of the American Academy of Audiology 14: 545-55.

[12] Valente, M., Abrams, H., Benson, D., Hnath-Chisolm, T., Citron, D., Hampton, D., Loavenbruck, A., Rickette, T., Solodar, H., and Sweetow, R. 2006. "Guidelines for the Audiologic Management of Adult Hearing Impairment." Audiology Today 18: 1-44.

[13] Ashoor, A. A., and Prochazka, Jr., T. 1982. "Saudi Arabic Speech Audiometry." Audiology 21: 493-508.

[14] Owens, E., Kessler, D. K., Telleen, C. T., and Schubert, E. D. 1981. "The Minimal Auditory Capabilities (MAC) Battery." Hearing Aid Journal 34: 9-34.

[15] Bakr, S. 1988. The Arabic Minimal Auditory Capabilities Test (M.D. Thesis). Ain Shams University, Egypt.

[16] King Abdulaziz University Hospital (KAUH). 2004. Arabic lip reading test (Unpublished materials).

[17] Gatehouse, S. 1999. "Glasgow Hearing Aid Benefit Profile: Derivation and Validation of a Client-centred Outcome Measure for Hearing Aid Services." Journal of the American Academy of Audiology 10: 80-103.

[18] Alkhamees, A. H. 2013. An Investigation of a Rehabilitation Training Programme to Facilitate the Benefits of Hearing Aid Use for Hearing Impaired Adults in Saudi Arabia (Unpublished PhD thesis). University of Surrey, United Kingdom.

[19] Norman, M., George, C., Downie, A., and Milligan, J. 1995. "Evaluation of a Communication Course for New Hearing Aid Users.” Scandinavian Audiology 24: 63-9.

[20] Jennings, M. B. 2009. "Clinical Report: Evaluating the Efficacy of a Group Audiologic Rehabilitation Program for Adults with Hearing Loss using a Goal Attainment Scaling Approach." Canadian Journal of Speech-Language Pathology and Audiology 33: 146-53.

[21] Taylor, K. S. 1993. "Self-perceived and Audiometric Evaluations of Hearing Aid Benefit in the Elderly." Ear and Hearing 14: 390-4.

[22] Hawkins, D. B. 2005. "Effectiveness of Counseling-based Adult Group Aural Rehabilitation Programs: a Systematic Review of the Evidence." Journal of the American Academy of Audiology 16: 485-93.

[23] Mulrow, C., Tuley, M. R., and Aguilar, C. 1992. "Sustained Benefits of Hearing Aids." Journal of Speech and Hearing Research 35: 1402-5.

[24] McArdle, R., Chisolm, T. H., Abrams, H. B., Wilson, R. H., and Doyle, P. J. 2005. "The WHO-DAS II: Measuring Outcomes of Hearing Aid Intervention for Adults." Trends 
in Amplification 9: 127-43.

[25] Wood, S. A., and Lutman, M. E. 2004. "Relative Benefits of Linear Analogue and Advanced Digital Hearing Aids." International Journal of Audiology 43: 144-55.

[26] Arlinger, S., Billermark, E., Oberg, M., Lunner, T., and Hellgren, J. 1998. "Clinical Trial of a Digital Hearing Aid.” Scandinavian Audiology 27: 51-61.

[27] Boothroyd, A. 2007. "Adult Aural Rehabilitation: What is it and does it Work?" Trends in Amplification 11: 63-71.

[28] Sweetow, R. W., and Sabes, J. H. 2006. "The Need for and Development of an Adaptive Listening and Communication Enhancement (LACE) Program.” Journal of the American Academy of Audiology 17: 538-58.

[29] Erber, N. P. 1975. "Auditory-visual Perception of Speech." Journal of Speech and Hearing Disorders 40: 481-92.

[30] Hutton, C. 1959. "Combining Auditory and Visual Stimuli in Aural Rehabilitation." Volta Review 61: 316-9.

[31] Siegenthaler, B. M., and Gruber, V. 1969. "Combining Vision and Audition for Speech Reception." Journal of Speech and Hearing Disorders 34: 58-60.

[32] Ewertsen, H. W., Birk, N. H., and Scott, N. S. 1970. "Audio-visual Speech Perception a Preliminary Report." Acta Oto-laryngologica 69: 229-30.

[33] Dalebout, D. 2009. The Praeger Guide to Hearing and Hearing Loss: Assessment, Treatment, and Prevention. London: Praeger.
[34] Barnett, S. 2002. "Communication with Deaf and Hard-of-hearing People: a Guide for Medical Education.” Academic Medicine 77: 694-700.

[35] Chen, Y., and Hazan, V. 2009. "Developmental Factor and the Non-native Speaker Effect in Auditory-visual Speech Perception." Journal of the Acoustical Society of America 126: 858-65.

[36] Walden, B. E., Erdman, S. A., Montgomery, A. A., Schwartz, D. M., and Prosek, R. A. 1981. "Some Effects of Training on Speech Recognition by Hearing Impaired Adults." Journal of Speech and Hearing Research 24: 207-16.

[37] Munro, K. J., and Lutman, M. E. 2004. "Self-reported Outcome in New Hearing Aid Users over a 24-week Post-fitting Period." International Journal of Audiology 43: 555-62.

[38] Cox, R. M. 1996. "The Abbreviated Profile of Hearing Aid Benefit (APHAB) Administration and Application." Phonak Focus 21.

[39] Cox, R. M., and Alexander, G. C. 1995. "The Abbreviated Profile of Hearing Aid Benefit." Ear and Hearing 16: 176-86.

[40] Kemker, B. E., and Holmes, A. E. 2004. "Analysis of Pre-fitting versus Post-fitting Hearing Aid Orientation using the Glasgow Hearing Aid Benefit Profile (GHABP)." Journal of the American Academy of Audiology 15: 311-23. 